\title{
Exploring Student Reasoning and Representation Construction in School Science Through the Lenses of Social Semiotics and Interaction Analysis
}

\author{
Erik Knain $^{1} \cdot$ Tobias Fredlund $^{1,2}$ (D) Anniken Furberg ${ }^{1}$
}

Accepted: 30 October 2020 / Published online: 5 January 2021

(C) The Author(s) 2021

\begin{abstract}
The meaning-making practices of science are multimodal and include representational forms such as spoken and written language, diagrams, graphs, equations, and images. Science learning proceeds through an ever-increasing grasp of such resources. This study aims at providing insight into how a combination of Interaction Analysis (IA) and social semiotic analysis can provide a deeper understanding of students' engagement and learning with science representations. Social semiotics offers an analytical lens and categories for interpreting nuances of meaning in the visual domain. IA places a strong methodological emphasis on grounding analysis in unfolding interactions among peers, teachers, and artefacts. Investigating a teaching design where students iteratively produce multimodal drawings of the greenhouse effect, we used a multimodal analysis of the students' drawings and an IA of transcribed video recordings of students' interactions with each other and their teacher. The analyses show a progression towards more scientific student drawings over the course of a lesson. This progression was made possible by sustained attention to critical details in the drawings, and the drawings and interactions were instrumental in developing a more sophisticated understanding of the mechanisms behind the greenhouse effect. IA provides important analytical insights into students' interest in the situation, which is at the heart of social semiotics. Social semiotics offers insight into the nuances of students' interpretations of the world and how they relate to the practices of disciplinary science.
\end{abstract}

Keywords Student-produced representations · Everyday naturalism · Scientific abstraction · Social semiotics · Interaction Analysis

Supplementary Information The online version contains supplementary material available at https://oi. org/10.1007/s11165-020-09975-1.

Erik Knain

erik.knain@ils.uio.no

1 Department of Teacher Education and School Research, University of Oslo, Oslo, Norway

2 Faculty of Education and Business Studies, University of Gävle, Gävle, Sweden 


\section{Introduction}

The meaning-making practices of science are multimodal and include representational forms such as spoken and written language, diagrams, graphs, equations, and images (Christianson 2014; Lemke 1998). Science learning therefore proceeds through an everincreasing grasp of such modes of meaning-making (Norris and Phillips 2003). Two major trends in socioculturally oriented research on student learning with representations focus on how students use representations to make science meaning (e.g. Jewitt et al. 2001) and on students' interactions related to the representations in use (e.g. Krange and Arnseth 2012). In this article, we bring these foci together to capture the development of students' reasoning with representations. To accomplish this, we craft a methodological approach informed by social semiotics (Kress and van Leeuwen 2006) and Interaction Analysis (IA; Jordan and Henderson 1995). Social semiotics offers an analytical lens and categories for interpreting nuances of meaning in the visual domain. IA places a strong methodological emphasis on grounding analysis in unfolding interactions among peers, teachers, and artefacts.

To recognise meaning-making as essentially multimodal is fundamental in social semiotics. For example, Tang et al. (2011) investigated students' use of various representational formats or modes to make meaning around the work-energy concept. They focused on the actions of the moment where representations are shaped and reshaped to accomplish new actions and meanings by analysing 'how the different modalities used at different moments of the students' interaction constitute the contextual configurations that shape the subsequent meanings they make' (Tang et al. 2011, p. 1780). The analysis of Jewitt et al. (2001) did not focus on student interaction but on students' texts, comprising a number of different modes. This resulted in a description of the students' meaning-making processes as transformations - from something mundane (an onion) to something more scientific (many onion cells) and as transductions from one mode (e.g. language) to another (e.g. drawing).

In IA, the interaction between teachers, students, and various artefacts (e.g. written texts or graphs) is analysed turn by turn to show how it affects students' learning processes (e.g. Krange and Arnseth 2012). Throughout these processes, different topics are sometimes set aside and taken up again later in the students' interactions. Learning processes sometimes involve students trying to interpret what different representations mean, such as a diagram showing how a solar panel works, a graph showing the change in $\mathrm{pH}$ level in a glass of water as the students blow air into it through a straw, or visual representations showing the function of a heat pump (Jornet and Roth 2015; Ingulfsen et. al. 2018). During such discussions, the diagram can function as a 'structuring' or 'deictic resource' that the students can talk about and point to. The IA of Furberg et al. (2013) also illustrates other functions characteristic of representations (see Ainsworth 1999), such as one diagram complementing or constraining the potential meaning of another.

Our contribution attempts to make explicit how using social semiotics and IA together provides an even richer insight into classroom interaction and students' engagement with science representations. This is in line with Halliday's (1993) claim that children are born with a disposition to make ideational meaning and enact relationships with others and that 'signs are created in the intersection of these two 
modes of activity' (p. 95). The following methodologically oriented research question guides the methodological focus of this article:

How can IA and social semiotics as a combined methodological lens provide new insights into students' conceptual sense making in their engagement with self-produced representations?

\section{Social Semiotics: Analysing Meaning-Making in the Visual Domain}

Social semiotics deals with meaning-making in all its forms. As a research field, it has many ancestors and lines of influence. An interesting way of approaching social semiotics is presented by Hodge and Kress (1988), who portrayed social semiotics as emanating out of the trash bin of the linguist Saussure, who treated it as outside his science of language. Social semiotics would then include culture, society, and politics as intrinsic to semiotics; other semiotic systems alongside verbal language; time, history, process, and change; the process of meaning-making; structures of the signified; and the material nature of signs (Hodge and Kress 1988, p. 18).

According to social semiotics, the basis for meaning-making is a person's situationally constituted interest (Kress 2010). A person's interest in making a representation is related to their cultural, social, and psychological history (Kress and van Leeuwen 2006). Because a representation can never fully represent every aspect of something but only parts thereof (Kress 2010), meaning-making always involves selecting what should and should not be included in a representation. In the science classroom, it is thus the students' interest at any given moment that determines what they consider the most appropriate modes of meaningmaking and what they decide to include in their own representations. Interest manifests as meaning-making events conditioned by the available resources and social structures involving power. In sum, meaning-making is always partial and conditioned; given the possibilities and restrictions of the modes involved, the meaning maker chooses the critical aspects to be represented in the moment (Kress and van Leeuwen 2006). Next, we provide brief accounts of the elements of social semiotics used in our analysis: mode, framing, foregrounding, and coding orientation.

Mode Social semiotic analyses differentiate between representational formats, or modes. Kress (2013, p. 60) describes mode as a 'socially shaped and culturally given resource for meaning-making'. Language and drawing are two examples of modes. Different modes have different possibilities or 'affordances' for meaning-making (Bezemer and Kress 2010, p. 14). For example, images do not have the same affordance as written text, although there might be some overlap. Images are better for showing what something looks like, whereas written text is better for naming things (see Kress 2010).

Framing The reading of scientific representations also depends on their framing. Framing refers to 'the boundaries to interpretation' (Kress 2013, p. 73). In multimodal text, the frame is often constituted by punctuation marks or physical space, such as the page of a book (Bezemer and Kress 2010). Lines in a drawing can separate an object from its surroundings (Kress and van Leeuwen 2006), whereas their absence may connect different objects. 
Foregrounding Another central construct is the notion of foregrounding. In a representation, such as an image, some parts will be foregrounded (Kress and van Leeuwen 2006). Foregrounding can be achieved in different ways in different modes. An image in a book, for example, can be brought to the fore by allotting it a larger part of the page (Kress and van Leeuwen 2006; Unsworth 1999). In printed writing, something can be foregrounded through its relative position in a sentence or the use of italics. Appropriate mode selection can emphasise some aspects of the content while other, perhaps more peripheral, content recedes to the background (see Kress et al. 1998).

Coding Orientation Another aspect of Kress and van Leeuwen's (2006) toolbox for analysing meaning-making that is useful for our analysis is coding orientation, which is related to Bernstein's (2003) interpretive codes. Coding orientation refers to the norms and expectations in a particular culture for how to present something, spanning a continuum from preferred to not preferred in terms of different aspects of meaningmaking. Coding orientation frames interpretations of what counts as real in a culture and is thus a cultural code. For example, a truthful image according to a culture endorsing a naturalistic coding orientation is balanced between most colour and no colour. In such cultures, images are therefore interpreted as more or less true against this standard. By contrast, technical and distilled meaning is considered truthful in cultures endorsing a technological coding orientation (Kress and van Leeuwen 2006), where the aspect of colour is often seen as less important. For the purposes of this paper, we refer to the latter coding orientation as scientific. As we analyse students' meaning-making, we consider whether the representations they produce would be deemed more 'true' or 'real' according to one or the other coding orientation.

\section{Interaction Analysis: Scrutinising Students' Conceptual Sense-Making Processes when Engaging with Visual Representations}

IA is an interdisciplinary method with roots in research fields such as ethnography, sociolinguistics, ethnomethodology, conversation analysis, and sociocultural theories (Jordan and Henderson 1995). Our use of IA procedures is based on a sociocultural perspective on learning; we regard learning as dynamic and dialogical meaning-making processes between interlocutors (Linell 2009). A central assumption in sociocultural perspectives is that all human interaction is mediated by cultural and semiotic tools (Vygotsky 1978). Language is considered the most important tool, providing a 'social mode of thinking' in social practices (Mercer 2004). Beyond talk and interaction, learning is contingent on material artefacts that inscribe and store knowledge and social practices (Vygotsky 1978). This implies that various material tools are conceived as mediational means that become integrated into students' meaningmaking processes. In the context of science education, visual representations, such as the canonical and student-constructed representations at the centre of the educational setting analysed in this article, can play an important role as mediating artefacts in students' appropriation of scientific knowledge and practices.

With these theoretical premises as a backdrop, we now turn to the practical analytical guidelines of IA. IA is a suitable method for investigating 'human activities, such as talk, nonverbal interaction, and the use of artefacts and technologies, identifying routine practices 
and problems and the resources for their solution' (Jordan and Henderson 1995, p. 42). Our conceptualisation of IA is based on three significant aspects. First, the analytical focus is not only on talk-in-interaction but also on participants' engagement with objects and artefacts. Second, IA combines microlevel analysis and ethnographic data. Ethnographic data enable an understanding of how microlevel activities are part of locally situated contexts and institutional practices (Jornet and Roth 2015). This implies that the unit of analysis is the what, where, who, and/or when of learning (Säljö 2009). Third, the analytical focus is also on 'interaction trajectories', which capture how students participate in meaning-making activities over time (Ludvigsen et al. 2011). The concept of trajectories is important because it allows for the possibility of exploring how students orient themselves in different situations over time. In other words, it expands the moment-to-moment analysis of meaning-making and takes into account how continuity and change are constructed in interaction; talk and interaction allow topics and themes to be discussed, negotiated, and left behind, sometimes to be reintroduced later (Engle and Conant 2002).

When it comes to practical guidelines informing an IA procedure, 'bracketing out' the analyst's pre-existing theories and interpretations is a key analysis principle (Schegloff 1991). Another practical guideline is linked to the situated aspect of interaction and what Linell (2009) refers to as the notion of indexicality - the meaning of utterances and actions depends on the context within which they are performed. Hence, the analysis of interaction focuses on what is going on and what the participants make topical through their conversation and interaction. This ensures that the analytical descriptions are oriented towards the interactional achievements instead of what might take place in the individuals' minds or the predefined categories or theories defined by the researcher (Jornet and Roth 2015).

In sum, our IA approach enables us to show and explore how social processes (i.e. interaction between interlocutors), engagement with multimodal representations (i.e. canonical and self-produced illustrations of the greenhouse effect), and individuals' development of conceptual understanding are intertwined and occur over time. This analytical approach allows us to identify how each individual contributes to shared knowledge construction, as well as the teacher's way of supporting students' development of conceptual understanding.

\section{Combining Social Semiotics and Interaction Analysis}

Social semiotics and IA share some key ideas about language and interaction. First, language use in the situation must be interpreted in a rich context understood as an ecology of the materiality of the situation, the present and nonpresent, and what has been and what comes next, as inferred from participants' utterances. Second, meaning-making in the situation is created through patterns of meaning as meaning potentials to be drawn on and realised in the situation. Finally, the materiality of the situation and affordances of tools shape social interaction.

Social semiotics in the visual domain allows us to systematically analyse the nuances of meaning in students' drawings. These nuances would otherwise pass unnoticed as they develop over time. Different features of students' drawings are seen as indications of their interest and selections in social settings. IA grounds analysis in unfolding interactions among peers, teachers, and artefacts. It also prescribes that analysis be 
carried out with a keen eye on what is manifest in the interaction and with caution about inferring what participants think or feel.

\section{Research Design}

We collected data from three iterative drawing activities carried out in a science classroom where the students were asked to draw and explain the greenhouse effect based on an experiment that simulated this phenomenon (see Fig. 1). The demonstration provided firsthand experience of the greenhouse effect by comparing the temperature in a glass beaker containing an enhanced amount of $\mathrm{CO}_{2}$ with that in an identical beaker containing ordinary air. Both beakers were put under a strong work lamp (see Niebert and Gropengießer 2013).

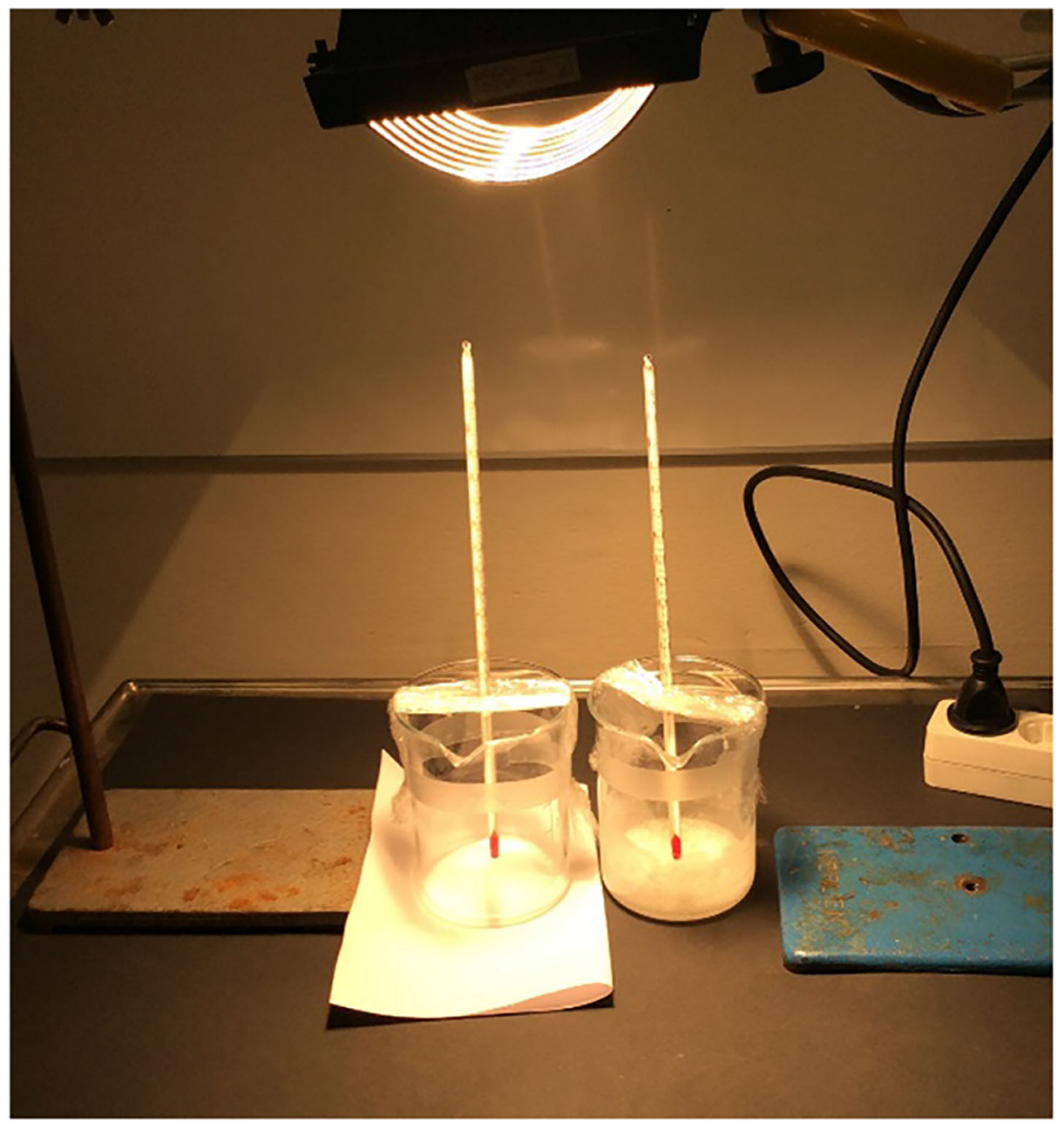

Fig. 1 The experimental setup 
The instruction, which was designed by a science teacher and the first and third authors, was implemented by the teacher in a class of twenty-five 15-16-year-old secondary school students. The course was the students' final compulsory science course in upper secondary school. The implementation took place during three school lessons (each lasting approximately 45-60 min) over the course of 2 weeks. During the first two lessons, the following activities took place: (1) a teacher-led brainstorming activity where the teacher wrote the students' ideas related to the greenhouse effect on the whiteboard, (2) a teacher-led introduction to the experiment, and (3) an iterative drawing activity. The third activity comprised two parts. In the first, drawings were produced by student dyads and revised to various degrees. In the second part, each student dyad was asked to merge their drawing with that of another dyad. We report on the first part of Activity 3 in this article, focusing on a student dyad that started over on new drawings four times.

The main data in this study consist of $10 \mathrm{~h}$ of transcribed video recordings of all studentteacher and student-student interactions that occurred during the three lessons constituting the greenhouse effect unit. Classroom interaction in three student focus groups was captured using head-mounted cameras, and additional video data were collected through a handheld video camera that recorded all student-teacher interactions during the class and groupwork activities. The video recordings were transcribed according to an adaptation of Jeffersonian transcription notations (Jefferson 1984). Table S1 in the Appendix explains the transcript notations. We translated the Norwegian conversations into English and used pseudonyms for the participants in the excerpts.

The student groups participating in the data collection were selected because they had volunteered to participate. We focus on one student dyad in one of the three focus groups for several reasons. First, the students in this dyad engaged in sustained inquiry by way of joint meaning-making and by continuously addressing issues and challenges that they found interesting during the drawing activity. Second, the dyad's capacity to explicitly verbalise their conceptual reasoning and explanations resulted in transparency in their meaning-making and decisions regarding how to represent the greenhouse effect. Third, the issues and challenges addressed by this dyad reflect the issues and challenges experienced by the other groups in the drawing activities. Thus, many features of the selected dyad's work could also be found in the other dyads' work.

In our analysis of the students' writing and drawing, we used the following social semiotic constructs: (1) the modes used by the students, (2) the framing of different units of meaning, (3) what was foregrounded in the students' meaning-making, and (4) the truthfulness of the students' drawing in terms of coding orientation. We also focused on changes in the students' orientations and on their choices in their iterative representational work (Kress and van Leeuwen 2006). The IA procedure applied in this article thus aims at providing an analytical account of the students' meaning-making processes in their interactions with each other and their teacher, as well as their self-produced representations as they engage in meaning-making processes. This entails an emphasis on what they present as significant in the activity and the educational setting in which they operate (Jordan and Henderson 1995).

\section{Results}

We follow students Knut and Ole as they draw and explain what is going on in the greenhouse effect simulation experiment. During the drawing activity, they used their laptops and 
textbooks as learning resources. All text in the students' drawings that we present has been translated from Norwegian into English.

\section{Episode 1: Drawing the First Version-'Setting the Scene'}

The students produced their first drawing based on the experiment. Just before this, the teacher had introduced the purpose of the experimental demonstration and how it constituted a simulation of atmospheric greenhouse processes. The students were urged to use the experimental demonstration when they drew and explained the greenhouse effect. Figure 2 shows Knut and Ole's first drawing.

Social Semiotic Analysis In Ole and Knut's first drawing, the sheet of paper by itself constitutes a frame for what is being drawn. As a material framing, the paper is familiar even to children who draw for leisure, so it is in no way special to the situation. What should be included in the drawing had to be drawn within that frame. The students' first drawing depicts three items - a sun and two beakers - centrally and symmetrically located on the page and with ample space around them. This simplistic drawing foregrounds the physical elements of the experimental setup while referring to the greenhouse effect occurring in the atmosphere (through the lamp drawn as the sun and the chemical formulae $\mathrm{CO}_{2}$ and $\mathrm{O}_{2}$ ).

Other physical elements, such as the table and thermometers, are excluded from Ole and Knut's first drawing. This suggests that, despite the drawing including only a few details, the students have undertaken significant semiotic work in selecting which aspects to include or exclude in their representation of the greenhouse effect experiment. With regard to the details, the beakers and sun are drawn schematically and thus somewhat less truthfully in terms of the naturalistic coding orientation. Still, the beakers are drawn

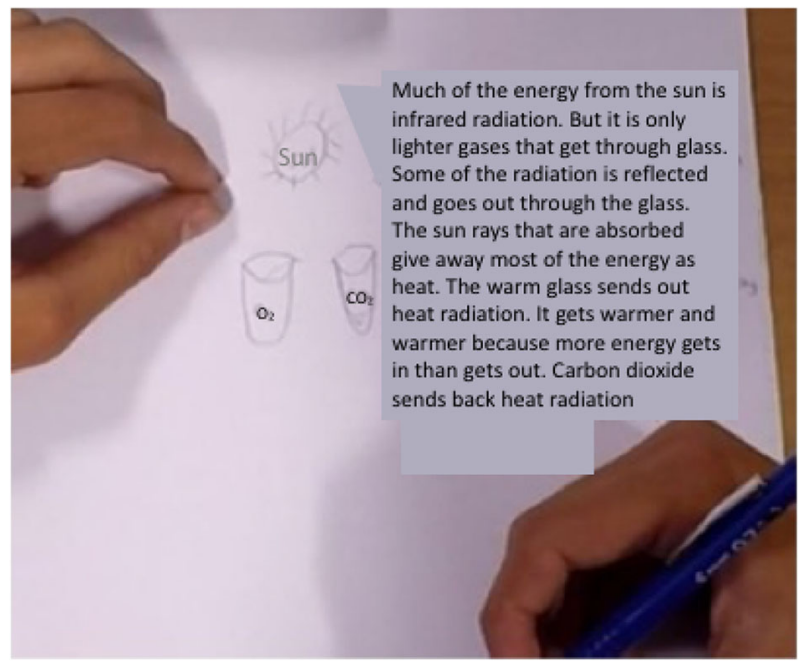

Fig. 2 Drawing 1 
as three-dimensional objects, which is highly regarded in this coding orientation. The students' illustration displays scientific abstraction only in the use of symbols for the chemical formulae of oxygen and carbon dioxide.

The modes used are drawing and writing (including the scientific symbolism). The modes are used according to their affordances: drawing for presenting spatial relationships and writing for naming things and describing what is happening (Bezemer and Kress 2010). The students' written text relies heavily on the textbook and creates a tension between the domains of the natural greenhouse effect, the demonstration experiment, and the textbook. The written text added by the students to their drawing represents entities and physical processes that cannot otherwise be seen in their drawing. Thus, there is a clear division of 'semiotic labour' (Matthiessen 2007, p. 37) between the written text and the drawing.

The interaction in Excerpt 1 occurred after Ole had written the accompanying text.

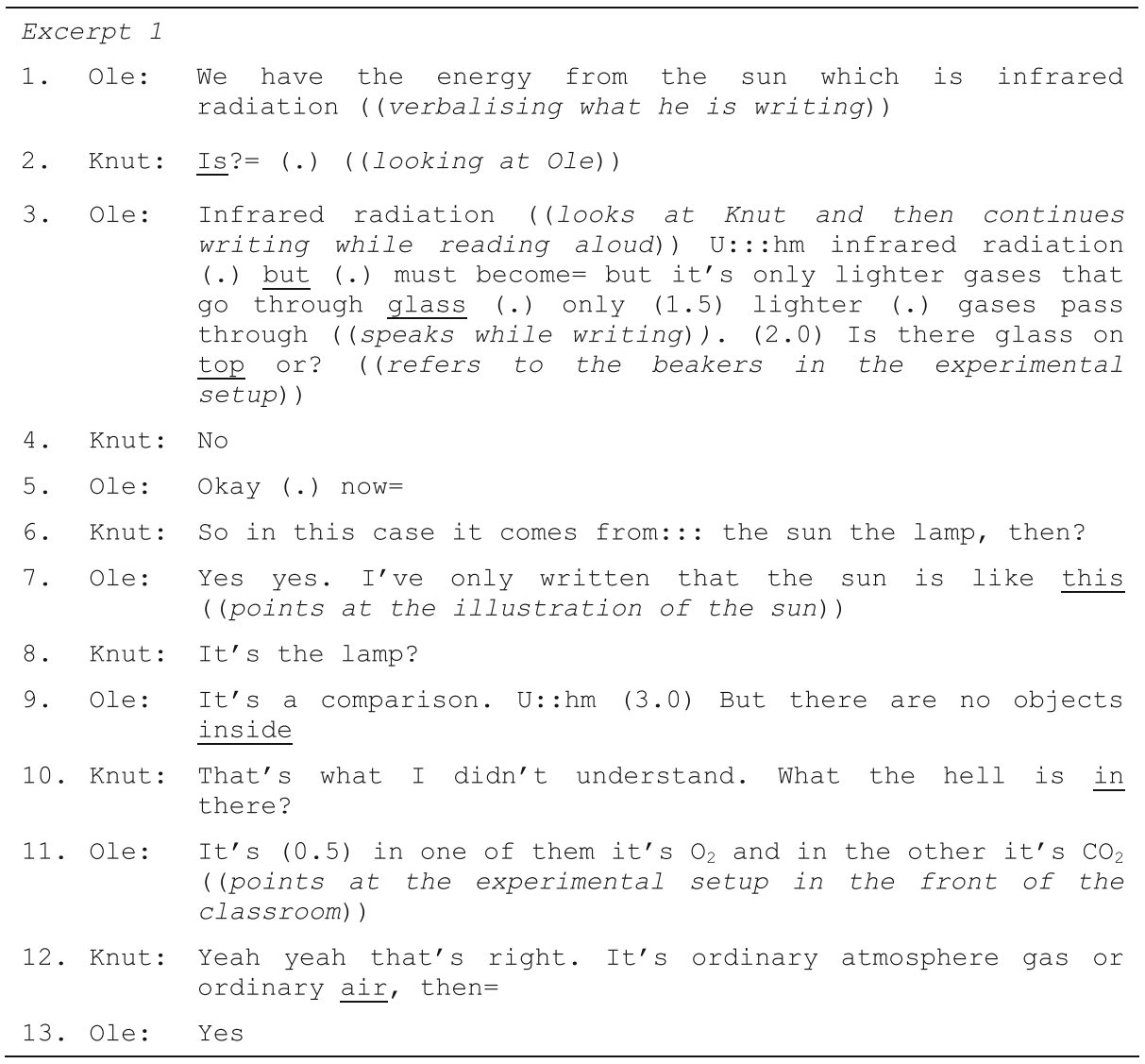

Interaction Analysis In this excerpt, sorting out the references to the natural greenhouse effect on the one hand (the sun is the light source) and the demonstration experiment (the lamp is the light source) on the other constitutes the theme of the conversation. The students use the terms 'sun' and 'lamp' interchangeably to make a 
connection between the experiment and the greenhouse effect. Ole uses the term 'sun' (line 1) and then adds 'lamp' (line 6). He then supports his terminology in line 9 by noting that 'it's a comparison' and then shifts his attention to what is happening inside the beakers. Knut reinforces this theme by noting that he 'did not understand' what is 'in there' (line 10). Ole replies that one beaker contains $\mathrm{CO}_{2}$ and the other contains $\mathrm{O}_{2}$ (line 11). From a disciplinary point of view, this is a crude distinction, as both beakers contain both gases but at different concentrations. The students relate their scientific explanation (lines 1-3), written to the right of the drawing, to the drawing itself. From a scientific point of view, however, the meaning that the students make is fragmented. Ole focuses on infrared radiation from the sun, but it is visible light that is important from a scientific point of view. There also seems to be some confusion between radiation and gas.

Synthesis The analyses of the students' drawing and their interaction in Excerpt 1 allow us to identify important shifts in the framing and foregrounding of the students' meaning-making. Due to the simplicity of the drawing, the sun and beakers are initially foregrounded. However, this foregrounding is somewhat ambiguous in that the students' drawn and verbal accounts include one item from the outside world (the sun) and two items from the demonstration experiment (the two beakers). The students address and negotiate this ambiguity in lines 6-9. The beakers are drawn in a way that indicates depth, which suggests that the students' attention is directed at these beakers and what goes on in this experiment.

\section{Episode 2: Drawing a Third Version-'Inside the Beakers'}

In Drawing 2 (Fig. 3), the students added more scientific detail than in Drawing 1, for example, by adding labelled arrows. After they had worked on their second drawing, Ole looked at a drawing by students from another group. He noticed that they had made a much larger drawing

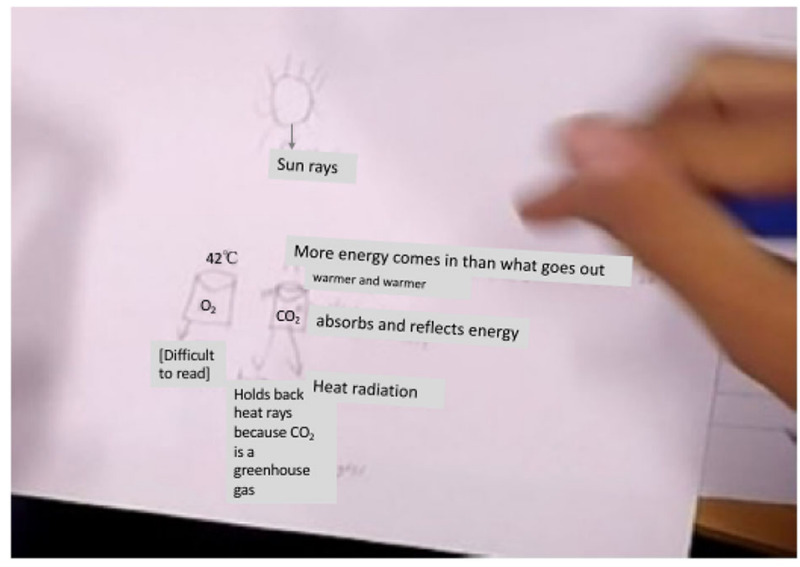

Fig. 3 Drawing 2 
and that they had included more details inside the beakers. Possibly inspired by their drawing, Ole decided to make a third drawing (see Fig. 4).

Social Semiotic Analysis In the third drawing, the lamp is drawn more naturalistically and looks more like a lamp than the sun. The beakers are simplified in that the threedimensionality is left out entirely and they are drawn as rectangles-again labelled $\mathrm{O}_{2}$ and $\mathrm{CO}_{2}$. The beakers are significantly larger than they were before. Ole draws arrows again but inside the beakers. Despite the lamp being drawn more naturalistically, Drawing 3 (Fig. 4) is less truthful than the previous drawings in terms of the naturalistic coding orientation. The truthfulness is instead increased in terms of the scientific coding orientation. In science representations, the presence of otherwise invisible items is to be expected, whereas contextual detail is not.

In the third drawing, the two beakers frame the physical processes portrayed by arrows. Thus, an inner framing has been created inside the outer framing (the sheet of paper). What is inside the beakers is brought to the fore. That the arrows directed upwards have a common origin at the bottom of the beakers suggests that something significant is happening there and makes the bottom visually salient. An explanatory text is also written between the beakers, suggesting that the radiation 'changes to a lighter radiation when it goes through glass'. Here, 'lighter radiation' presumably stands in contrast to the 'infrared radiation' and 'sun rays' that were written below the drawn lamp, but we do not know for sure what Ole meant by this. Furthermore, it says 'absorbs' at the bottom of the $\mathrm{CO}_{2}$ beaker and 'reflects' at the bottom of the $\mathrm{O}_{2}$ beaker. Thus, the mode of written language still has the important role of adding specificity to the drawing. But the visual mode generally takes a more prominent role in the third drawing than it did in the first two.

To conclude, a shift in the semiotic labour between modes-from writing to drawing-took place from the second drawing to the third. In the latter, the affordance of drawing was used to indicate both the existence of radiation and the direction of its propagation. In accordance with the social semiotic framework, we interpret this as a shift in the students' interest taking place from the second to the

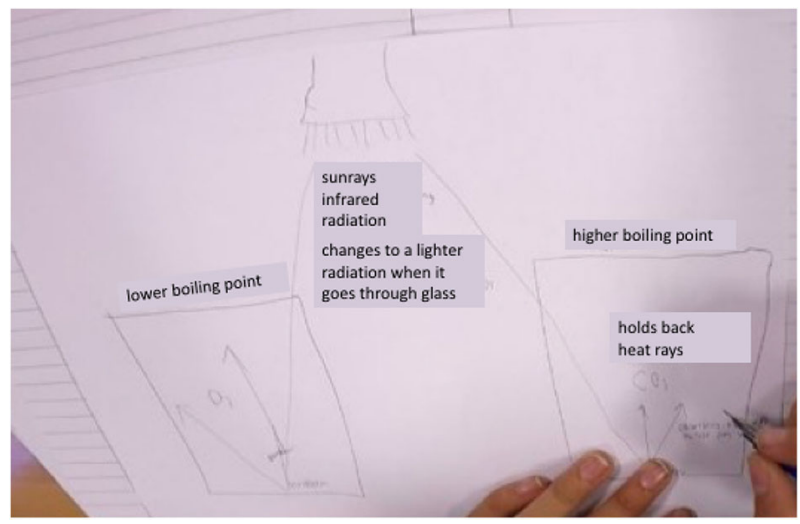

Fig. 4 Drawing 3 
third drawing: away from the material setting towards what is happening inside the beakers and, subsequently, to what is happening at the bottom of the beakers.

When the students had finished their third drawing, Knut summoned the teacher to ask him about the things taking place at the bottom of the beaker. In Excerpt 2, we enter the conversation as the teacher is using the analogy of radio waves from mobile phones to explain to the students what is happening in the $\mathrm{CO}_{2}$ beaker.

Excerpt 2

1. Teacher: If we look at= if we look at this wall it suggests that radio waves (.) can go through (1.0) go through walls (.) visible light cannot do that

2. Ole: No

3. Teacher: so there are different types of radiation that can go through different types of materials. Not all kinds of radiation go through all kinds of materials. So what happens $=(0.5)$ is that both visible light and heat radiation go through (.) oxygen and nitrogen (lpoints towards the left beaker in the boys' drawing)) it's transparent to them. But (.) when it comes to $\mathrm{CO}_{2}$ (lpoints inside the right beaker in the students' drawing)l and water vapour, methane, the important greenhouse gases. So they're transparent to visible light (lmoves his pencil across the drawing from above to the bottom of the beaker)) but they are not as transparent to heat radiation ((points at the centre of the right beaker with his pen)) so it's absorbed and sent back again down to the earth ((moves his pen quickly above the students' drawing from the centre to the bottom of the right beaker))

4. Ole:

Yes uhum we have written that

5. Teacher: So:: ((points with his pencil towards the text under the lamp in the students' drawing))

6. Knut: Okay so here ((points to the left beaker with his pencil)) both go through and heat (.) while here (lpoints at the right beaker)) only visible goes through and not heat

7. Teacher: Some heat but not all (lpointing at the right beaker with his pen))

8. Knut: Not all (.) right

9. Ole: So some of it is absorbed

10. Teacher: So (.) how do we differentiate between the short-wave heat radiation $=$ no the short-wave radiation from the sun (lpoints with his pen at the line drawn from the lamp towards the right beaker)) and the long-wave heat radiation that is sent up from the ground ((tracing a line upwards from the bottom of the beaker))? Is there some way to show this in the drawing (.) that they're different? 
11. Knut: By (.) by making that one longer than the other? ((referring to the right beaker in a sweeping way))

12. Ole:

Yes that one is longer than the other (ltraces the line from the lamp into the right beaker with his index finger))

13. Teacher: Yes but this ( points at both endpoints of the line from the lamp to the bottom of the beaker)) only suggests that it has travelled farther

14. Ole: Yes the waves are ((shows the shape of a wave in the air with his index finger)) when wave crests and such things but should we continue like this?

15. Teacher: Yes (.) is there some way to show...?(.) How does the textbook show waves?

16. Knut: (lopens the page in the textbook displaying a greenhouse effect diagram))

17. Knut: U:::hm (.) like this (.) it's not something like::: that:: ( (points at the waves in the textbook diagram. See Figure 5))

18. Teacher: Is it possible to see a difference? ((points at the textbook figure))

19. Ole: ((points in the figure in the textbook.)) Yes the wavelength is longer and the other is shorter

20. Knut: Then perhaps we should make= ((points quickly at the right beaker in the drawing)) okay (.) I understand it

21. Ole Yes

22. Teacher: It's wise to have $a=($.$) to think that the figure should$ be= like if I hadn't heard about this before I could take a look at it and understand what's going on

23. Ole

24. Teacher

25. Ole:
Yes okay

And in this ((points at the drawing)) I can't see the difference between short-wave and long-wave radiation. I can't really see what's happening to the $\mathrm{CO}_{2}$ in the atmosphere ((points inside the right beaker)) to the heat radiation when it meets the $\mathrm{CO}_{2}$ in the atmosphere No

Interaction Analysis In Excerpt 2, the teacher's account of different gases that are transparent to different types of radiation is an account of key elements in an explanation of the greenhouse effect (line 3). The teacher ties his account to the students' drawing by the mode of gestures - pointing at specific locations with his pen and moving the pen across the drawing. Ole affirms the teacher's account by saying 'we have written that' (line 4). Knut then rephrases the teacher's account, connecting it to the drawing and noting the differences between the two beakers (line 6). An important development follows in line 10, when the teacher asks the students how the difference between short-wave and long-wave radiation can be shown in the drawing. In doing so, he shifts the focus from the represented explanation to a metarepresentational perspective on the act of representing (see diSessa 2004). Again, he uses 
gestures to tie his questioning to the students' drawing. In line 12, Ole suggests that the difference in wavelength is shown by the different lengths of the lines; the teacher then offers a different interpretation of Ole's suggestion - that the lengths of the arrows mean they have travelled different distances. In this way, he gently dismisses Ole's explanation. The teacher picks up on Ole's use of the word 'waves', suggesting that the students look at the textbook's way of showing waves (line 15). Figure 5 shows the image from the textbook.

In lines 16-19, the students study the textbook image. The teacher then asks them to note the difference between the arrows in the textbook figure (line 18). Ole then states the important distinction between longer and shorter wavelengths (line 19). In Fig. 5, there is a consistent variation between the short-wave radiation from the sun and the long-wave radiation from the earth's surface. Knut then makes a connection to their drawing by pointing and affirms that 'I understand it' (line 20). In line 24, the teacher points out that the current version of the students' drawing does not signify the difference between short- and long-wave radiation, and he advises the students to include that element in their drawing.

Synthesis In this excerpt, the teacher referred to items in the students' drawing by means of deictic terms ('that one'); more importantly, he used gestures to tie the students' drawing to the analogy of radio waves that can go through walls. In other words, the drawing functioned as a structuring resource around which the speech and gestures used in the students' discussion with the teacher were coordinated (see also Fredlund et al. 2012).

The situation arose as Knut asked the teacher what happens at the bottom of the beakers. This focus of attention had been developing gradually until this point. The student-teacher interaction was key to the next step of developing their drawing. But the work of implementing the outcome of this interaction into a revised account still needed to be done. Episode 3 illustrates this work.

\section{Episode 3: 'Towards the Mechanism Behind the Greenhouse Effect'}

The teacher-directed dialogue in Episode 2 led the students to draw a fourth drawing. This time, Knut drew. Both the student interaction and the fourth drawing show that the students

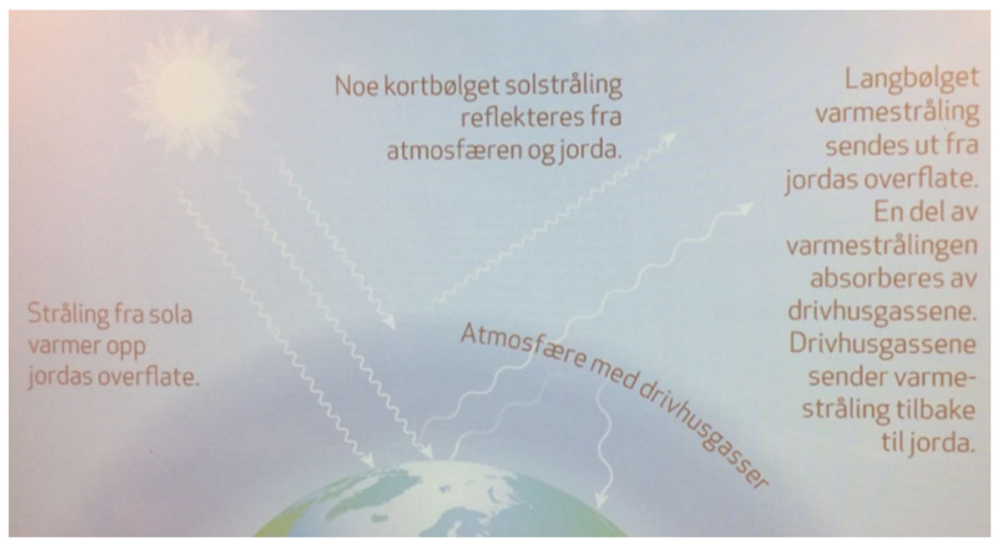

Fig. 5 The greenhouse effect diagram in the textbook (van Marion et al. 2013, p. 112). Permission to reproduce this figure has been granted by Gyldendal and the artist 
used the teacher's explanation, the textbook, their previous drawings, and the experimental setup as resources in the process of designing their final drawing. Their interactions and the drawing show how the students continued to explore the affordance of the mode of drawing in a more scientific way.

Social Semiotic Analysis In the fourth drawing (Fig. 6), the beakers and lamp continue to function as a frame for aspects of the experiment that the students found more interesting and are therefore foregrounded. This time, the beakers are drawn with a different drawing technique, which renders them fainter. The result is that the demonstration setup recedes further into the background. This background role of the beakers is further strengthened by the fact that the right beaker is partly overwritten by an explanation at the top of the righthand side of the paper. The aspects of the greenhouse effect that are foregrounded in this drawing are radiation and $\mathrm{CO}_{2}$ molecule. Another aspect of the experiment shown in the drawing is the different temperatures that the students read from the thermometers inserted into the beakers.

The wavy arrows representing different types of radiation are the salient items in the drawing. The drawing and written text are complementary in that the written language describes how the beaker containing $\mathrm{CO}_{2}$ gets warmer as the ' $\mathrm{CO}_{2}$ glass' holds back more heat radiation; the drawing illustrates this by showing three heat arrows leaving the beaker labelled ' $\mathrm{O}_{2}$ ' and only one leaving the $\mathrm{CO}_{2}$ beaker. In Drawing 4, the distinction between different wavelengths of radiation is made through the drawing of wavy lines similar to that of the textbook (see Fig. 5). This allows the students to explicate where the change from short waves to long waves occurs (even if the location they suggest in their drawing in Fig. 6 is not fully correct from a scientific point of view).

In terms of the division of semiotic labour, the mode of writing has a complementary function to that of drawing in that the bullet points explain what is drawn and use a number of technical terms (e.g. 'radiation' and ' $\mathrm{CO}_{2}$ molecule'). The scientific detail is also increased

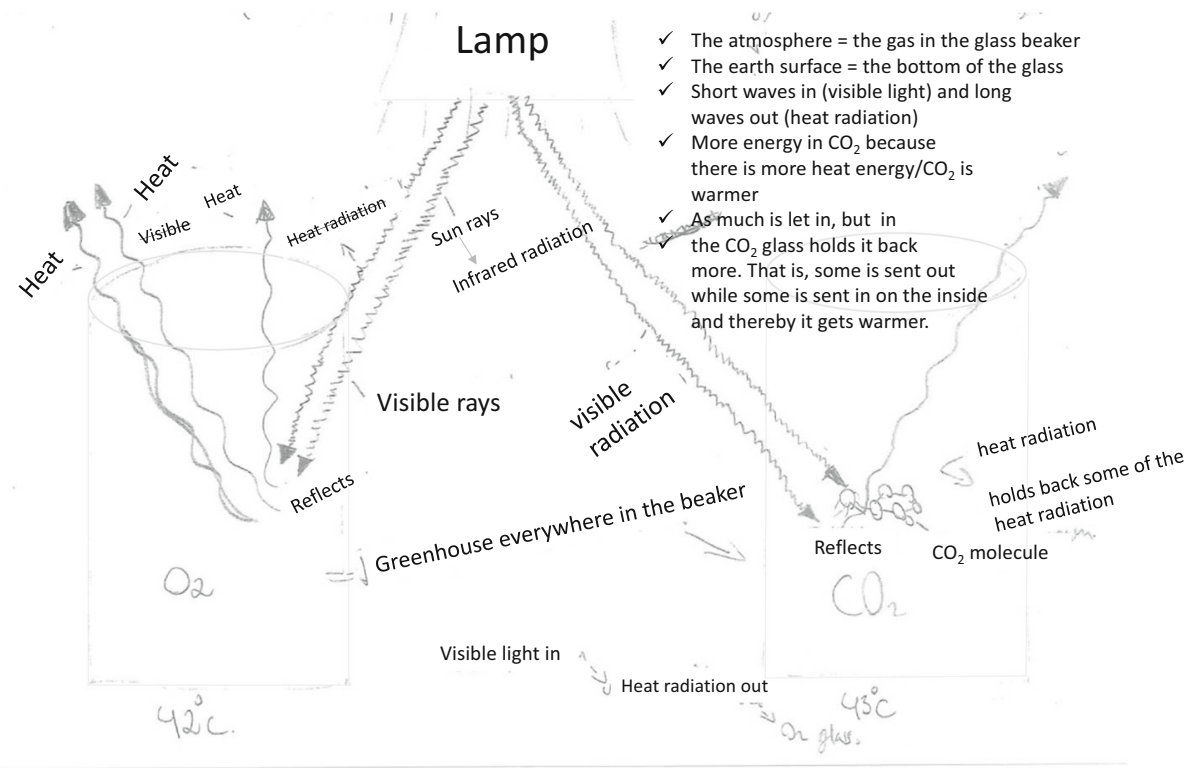

Fig. 6 Drawing 4 (the outlines of the beakers have been enhanced for visibility) 
considerably in the mode of drawing. The truthfulness is therefore increased in terms of the scientific coding orientation but lowered in terms of the naturalistic one-except that the beakers have again been drawn three-dimensionally.

\section{Discussion}

Our analyses of the episodes show a progression in the students' drawings during the classroom activity. The drawings initially depicted what the students could see but left out important aspects of what occurred in the experiment. The drawings and discussions were originally of an everyday character. Later, the drawings and language focused more on otherwise invisible scientific aspects. From the start of the students' work, the domain knowledge was written and separated from the drawings, but it was later gradually integrated as clusters of drawn and written items (see Baldry and Thibault 2006). The progression towards scientific abstraction can be identified and followed through the analytical categories of mode, framing, foregrounded items, and coding orientation (see Table 1).

Our semiotic analyses show that Ole and Knut's explanatory drawings developed from focusing on the experimental setup towards focusing on what happened inside the beakers. In the first drawing, the lamp was drawn and labelled as the sun, and two beakers were drawn and labelled ' $\mathrm{O}_{2}$ ' and ' $\mathrm{CO}_{2}$ ', respectively. In the second drawing, labelled arrows were introduced. In the third drawing, larger beakers were drawn to create a frame for a sketch of what occurred inside them. In the final drawing, the beakers were partly overwritten by explanatory text, and short- and long-wave arrows were labelled with 'visible radiation' and 'heat radiation', respectively.

A significant shift took place in this sequence of drawings in terms of framing and what was foregrounded. Initially, the experimental setup was foregrounded in the mode of drawing (although the lamp was drawn as the sun, indicating that the students were aware of the analogy between the lamp and the sun). What was foregrounded in Drawing 4 was what the students labelled visible radiation and heat radiation, which were drawn as waves of different wavelengths. These shifts in foregrounding were closely connected to shifts in framing. The blank piece of paper initially functioned as a frame for the drawn beakers (Drawing 1). Later, the beakers became the frame for the processes that were drawn as occurring inside them (Drawing 3). Written language was initially used in an everyday manner (e.g. to label the sun), but a shift later took

Table 1 Overview of the findings focusing on the visual mode

\begin{tabular}{|c|c|c|c|c|}
\hline Drawing & Mode & Framing & $\begin{array}{l}\text { Foregrounded } \\
\text { items }\end{array}$ & $\begin{array}{l}\text { Coding } \\
\text { orientation }\end{array}$ \\
\hline 1 & $\begin{array}{l}\text { Drawing and writing separated. } \\
\text { Supported by spoken language. }\end{array}$ & $\begin{array}{l}\text { Sheet of } \\
\text { paper }\end{array}$ & Beakers and sun & $\begin{array}{l}\text { Mostly } \\
\text { naturalistic }\end{array}$ \\
\hline 2 & $\begin{array}{l}\text { Drawing with labels and arrows } \\
\text { suggesting what is happening inside. } \\
\text { Supported by } \\
\text { spoken language. }\end{array}$ & $\begin{array}{l}\text { Sheet of } \\
\text { paper }\end{array}$ & Beakers and sun & $\begin{array}{l}\text { Mostly } \\
\text { naturalistic }\end{array}$ \\
\hline 3 & $\begin{array}{l}\text { Drawing dominating, with writing } \\
\text { for annotation and short descriptions. }\end{array}$ & $\begin{array}{l}\text { Sheet, } \\
\text { experiment }\end{array}$ & $\begin{array}{l}\text { Beakers (bottom) } \\
\text { and rays }\end{array}$ & $\begin{array}{l}\text { Both naturalistic } \\
\text { and scientific }\end{array}$ \\
\hline 4 & $\begin{array}{l}\text { Drawing with annotation, bullet } \\
\text { point explanation, and flow chart. }\end{array}$ & $\begin{array}{l}\text { Sheet, } \\
\text { experiment }\end{array}$ & $\begin{array}{l}\text { Different types } \\
\text { of radiation }\end{array}$ & Mostly scientific \\
\hline
\end{tabular}


place towards the use of more technical terms in the students' meaning-making. Part of this shift was an increased amount of detail in the students' drawing, which, together with the narrowing of the frame, 'zoomed in' on the salient features of the greenhouse effect.

The students' social interaction, as well as their engagement with the canonical and studentproduced representations, constituted a driving force behind this development towards a more complex and abstract explanation. As a result, the drawing shifted from showing everyday attributes in an ordinary manner (the beakers and the sun) to illustrating aspects of a scientific explanation of the greenhouse effect (wave-shaped arrows and scientific terms such as radiation, energy, reflection, and absorption) in a more scientific manner. The changes that occurred during the lesson in the language, in what was foregrounded in the drawing, and in the framing of the drawing suggest that a shift in coding orientation took place: The realness and truthfulness increased in terms of the scientific coding orientation but diminished in terms of the naturalistic one.

Paying close attention to what students say in the moment-to-moment interaction of their interaction trajectories - which is at the heart of IA - can reveal how their interest in a situation emerges and develops (Jornet and Roth 2015). In this study, we followed a well-functioning student group to illustrate our analytical contribution. It is important to keep in mind the role of the group dynamic; further research could apply the analytical approach presented here in various settings.

The IA provided important analytical insights into students' interest in the situation, which is at the heart of social semiotics. At the same time, the social semiotic analysis offered insights into the nuances of students' scientific interpretation of the world and how school activities relate to the practices of disciplinary science. Our study shows that students' development of a more technical and abstract understanding can be analysed as patterns of social interaction and visual representations. Further research could scrutinise other aspects of conceptual development in different knowledge domains and among students with diverse backgrounds. The framework presented in this article that enabled a description of the conceptual development could be used in teacher education to guide teachers on what to focus on in their assessment of students' work and to engage students in conversations that bridge students' beliefs and scientific concepts. What is certain is that students need plenty of time and support to produce different modes as they learn about complex scientific processes and work their way from everyday naturalism towards scientific abstraction.

Funding Funding from the Research Council of Norway, grant 249872, is gratefully acknowledged.

Open Access This article is licensed under a Creative Commons Attribution 4.0 International License, which permits use, sharing, adaptation, distribution and reproduction in any medium or format, as long as you give appropriate credit to the original author(s) and the source, provide a link to the Creative Commons licence, and indicate if changes were made. The images or other third party material in this article are included in the article's Creative Commons licence, unless indicated otherwise in a credit line to the material. If material is not included in the article's Creative Commons licence and your intended use is not permitted by statutory regulation or exceeds the permitted use, you will need to obtain permission directly from the copyright holder. To view a copy of this licence, visit http://creativecommons.org/licenses/by/4.0/.

\section{References}

Ainsworth, S. (1999). The functions of multiple representations. Computers \& Education, 33(2-3), $131-152$. Fredlund, T., Airey, J., \& Linder, C. (2012). Exploring the role of physics representations: an illustrative example from students sharing knowledge about refraction. European Journal of Physics, 33(3), 657-666. 
Furberg, A., Kluge, A., \& Ludvigsen, S. (2013). Student sensemaking with science diagrams in a computerbased setting. International Journal of Computer-Supported Collaborative Learning, 8(1), 41-64.

Ingulfsen, L., Furberg, A., \& Strømme, T. . Aa. . (2018). Students' engagement with real-time graphs in CSCL settings: scrutinizing the role of teacher support. International Journal of Computer-supported Collaborative Learning, 13, 365-390.

Baldry, A., \& Thibault, P. J. (2006). Multimodal transcription and text analysis: a multimedia toolkit and coursebook. London: Equinox.

Bernstein, B. (2003). Class, codes and control: the structuring of pedagogic discourse (Vol. 4). Routledge.

Bezemer, J., \& Kress, G. (2010). Changing text: a social semiotic analysis of textbooks. Designs for Learning, $3(1-2), 10-29$.

Christianson, S. (2014). 100 diagrams that changed the world. London: Batsford.

diSessa, A. A. (2004). Metarepresentation: Native competence and targets for instruction. Cognition and Instruction, 22(3), 293-331.

Engle, R. A., \& Conant, F. R. (2002). Guiding principles for fostering productive disciplinary engagement: explaining an emergent argument in a community of learners classroom. Cognition and Instruction, 20(4), 399-483.

Halliday, M. A. K. (1993). Towards a language-based theory of learning. Linguistics and Education, 5(2), 93116.

Hodge, R., \& Kress, G. (1988). Social semiotics. New York: Cornell University Press.

Jefferson, G. (1984). Transcription notation. In J. Atkinson \& J. Heritage (Eds.), Structures of social interaction (pp. ix-xvi). New York: Cambridge University Press.

Jewitt, C., Kress, G., Ogborn, J., \& Tsatsarelis, C. (2001). Exploring learning through visual, actional and linguistic communication: the multimodal environment of a science classroom. Educational Review, 53(1), 5-18.

Jordan, B., \& Henderson, A. (1995). Interaction analysis: foundations and practice. The Journal of the Learning Sciences, 4(1), 39-103.

Jornet, A., \& Roth, W. M. (2015). The joint work of connecting multiple (re)presentations in science classrooms. Science Education, 99(2), 378-403.

Krange, I., \& Arnseth, H. C. (2012). Students' meaning making in science: solving energy resource problems in virtual worlds combined with spreadsheets to develop graphs. Cultural Studies of Science Education, 7(3), 585-605.

Kress, G. (2010). Multimodality: a social semiotic approach to contemporary communication. London: Routledge.

Kress, G. (2013). What is mode? In C. Jewitt (Ed.), The Routledge handbook of multimodal analysis (pp. 60-75). London: Routledge.

Kress, G., \& van Leeuwen, T. (2006). Reading images: the grammar of visual design (2nd ed.). London: Routledge.

Kress, G., Ogborn, J., \& Martins, I. (1998). A satellite view of language: some lessons from science classrooms. Language Awareness, 7(2-3), 69-89.

Lemke, J. L. (1998). Multiplying meaning: visual and verbal semiotics in scientific text. In J. R. Martin \& R. Veel (Eds.), Reading science: critical and functional perspectives on discourses of science (pp. 87-114). London: Routledge.

Linell, P. (2009). Rethinking language, mind and world dialogically: interactional and contextual theories of human sense-making. Charlotte: Information Age.

Ludvigsen, S. R., Rasmussen, I., Krange, I., Moen, A., \& Middleton, D. (2011). Intersecting trajectories of participation: temporality and learning. In S. R. Ludvigsen, A. Lund, I. Rasmussen, \& R. Säljö (Eds.), Learning across sites: New tools, infrastructures and practices (pp. 105-121). New York: Routledge.

Matthiessen, C. M. I. M. (2007). The multimodal page: a systemic functional exploration. In T. D. Royce \& W. L. Bowcher (Eds.), New directions in the analysis of multimodal discourse (pp. 1-62). Mahwah, NJ: Lawrence Erlbaum Associates.

Mercer, N. (2004). Sociocultural discourse analysis: analysing classroom talk as a social mode of thinking. Journal of Applied Linguistics, 1(2), 137-168.

Niebert, K., \& Gropengießer, H. (2013). Understanding the greenhouse effect by embodiment - analysing and using students' and scientists' conceptual resources. International Journal of Science Education, 36(2), 277-303. https://doi.org/10.1080/09500693.2013.763298.

Norris, S. P., \& Phillips, L. M. (2003). How literacy in its fundamental sense is central to scientific literacy. Science Education, 87(2), 224-240.

Säljö, R. (2009). Learning, theories of learning, and units of analysis in research. Educational Psychologist, 44(3), 202-208. 
Schegloff, E. A. (1991). Conversation analysis and socially shared cognition. In L. B. Resnick, J. M. Levine, \& S. D. Teasley (Eds.), Perspectives on socially shared cognition (pp. 150-171). Washington, DC: American Psychological Association.

Tang, K. S., Tan, S. C., \& Yeo, J. (2011). Students' multimodal construction of the work-energy concept. International Journal of Science Education, 33(13), 1775-1804.

Unsworth, L. (1999). Explaining school science in book and CD ROM formats: using semiotic analyses to compare the textual construction of knowledge. International Journal of Instructional Media, 26(2), 159-179.

van Marion, P., Stolevik, E., Svendsen, B., Thyrhaug, T., Hov, H., \& Trongmo, Ø. (2013). Senit SF Naturfag (Senit SF Science). Oslo: Gyldendal.

Vygotsky, L. S. (1978). Mind in society-The development of higher psychological processes. Cambridge: Harvard University Press.

Publisher's Note Springer Nature remains neutral with regard to jurisdictional claims in published maps and institutional affiliations. 